\title{
Comprendiendo el Diseño Universal desde el Paradigma de Apoyos: DUA como un Sistema de Apoyos para el Aprendizaje
}

\author{
Understanding the Universal Design from the Support \\ Paradigm: UDL as a Support System for Learning
}

\author{
Victoria Sánchez-Gómez* \\ Mauricio López \\ Universidad de Chile, Chile
}

\begin{abstract}
Este artículo desarrolla una propuesta teórica consistente en la comprensión del diseño universal para el aprendizaje (DUA), propuesto por Meyer, Rose y Gordon (2014), como la planificación de un sistema de apoyos. Se justifica la necesidad de esta vinculación a partir de la importancia de que la planificación curricular logre responder efectivamente a las necesidades de todos los estudiantes del aula, comprendiendo estudiantes con y sin discapacidad. Si bien el DUA cuenta con amplio desarrollo conceptual y orientaciones metodológicas sobre su implementación, no se especifica en su formulación cómo se incorporan, teórica ni metodológicamente, las necesidades de los estudiantes en su planificación. Se propone vincular el DUA con el paradigma de apoyos, específicamente, mediante la comprensión de este como un sistema de apoyos para el aprendizaje, lo que trae consigo la crucial incorporación del constructo necesidades de apoyo. Este constructo ya es ampliamente utilizado en el ámbito de la discapacidad y, como constructo psicológico, dispone de avances psicométricos y metodológicos que permiten su evaluación. Finalmente, se justifica la necesidad de desarrollar un instrumento que evalúe las necesidades de apoyo, específicamente para el aprendizaje, que permita atender, desde la planificación del DUA, a las necesidades de todos y todas las estudiantes.
\end{abstract}

Descriptores: Planificación de la educación; Igualdad de oportunidades educativas; Educación universal; Diversificación de la educación; Educación integradora.

This article develops a theoretical proposal that consists in the understanding of the universal design for learning (UDL), proposed by Meyer, Rose and Gordon (2014), as the planning of a support system. The need for this link is justified based on the importance of curricular planning effectively responding to the needs of all students in the classroom, including students with and without disabilities. Although the UDL has broad conceptual development and methodological guidelines about its implementation, it is not specified in its formulation how the needs of students are incorporated theoretically or methodologically into its planning. It is proposed to link the UDL with the support paradigm, specifically, by understanding it as a support system for learning. This formulation brings with it the crucial incorporation of the support needs construct. This construct is already widely used in the field of disability and, as a psychological construct, it has psychometric and methodological advances that allow its evaluation. Finally, the need to develop an instrument that assesses the needs of support, specifically for learning, that allows to attend, from the planning of the UDL, to the needs of all and all students is justified.

Keywords: Educational planning; Equal opportunity in education; Universal education; Diversification of education; Inclusive education.

*Contacto: victoria.sanchez@uchile.cl

ISSN: 0718-7378

www.rinace.net/rlei/
Recibido: $\quad 30 / 12 / 2019$

$1^{\text {a }}$ Evaluación: 20/01/20202

Aceptado: 03/03/2020 


\section{Introducción}

Este trabajo surge del interés por estudiar los apoyos como parte de la elaboración conceptual de la contribución de la psicología y las ciencias del aprendizaje y del desarrollo de la inclusión educativa. En este trabajo entendemos inclusión educativa como el proceso mediante el cual el sistema educativo ofrece respuestas pedagógicas a las diferentes necesidades e identidades de los estudiantes, garantizando el derecho a la educación y el acceso al currículo por parte de todos. En este sentido, se ha especificado que, desde una perspectiva inclusiva, el acceso al currículo conlleva presencia, aprendizaje y participación (Ainscow, Booth y Dyson, 2006). Presencia implica que los estudiantes puedan estar, aprender y colaborar con otros; aprendizaje, en tanto, apunta a que todos los estudiantes puedan progresar en sus capacidades y desarrollen al máximo su potencial mediante experiencias educativas significativas que no apuntan únicamente al rendimiento académico (Ainscow y Miles, 2009); mientras que participación supone la oportunidad de implicarse en la toma de decisiones, reconocer y ser reconocidos y aceptados por quienes son (Ainscow, Both y Dyson, 2006; Albornoz, Silva y López, 2015) y participar en actividades extracurriculares, sociales y comunitarias (Amor et al., 2018). Esta aseveración no ha estado libre de tensiones. Norwich y Koutsouris (2017), por ejemplo, han examinado la tensión entre dos concepciones de inclusión que priorizan aspectos considerados como excluyentes: por una parte, la implicación en el aprendizaje, que antepone el aprendizaje independientemente de la localización, y por otra, la participación, que privilegia el que todos compartan los mismos espacios en el marco de la escuela regular. Sin embargo, la propuesta actual considera el aprendizaje y la participación de los estudiantes como elementos igualmente necesarios para la inclusión educativa, considerándose posible maximizar la participación de todos como objetivo curricular de tipo social.

Con respecto a la inclusión de personas con discapacidad en la escuela, en las últimas décadas se ha producido a nivel internacional un importante cambio el cual ha implicado un replanteamiento respecto del modo en que se comprende la discapacidad. Las perspectivas más actuales han transitado desde un énfasis en el déficit, a un énfasis en la adecuada modificación del contexto para mejorar el funcionamiento de estos estudiantes. El nuevo paradigma de discapacidad emergente correspondiente a una perspectiva ecológico-contextual se entiende en gran medida por dos modelos altamente implicados: el modelo multidimensional y el paradigma de apoyos. El modelo multidimensional de funcionamiento humano comprende la discapacidad desde el contexto y las interacciones que ahí suceden, y no como una característica intrínseca de la persona, otorgando un rol central al papel que juegan los apoyos en la mejora del funcionamiento. Por su parte, el paradigma de apoyos, altamente implicado en el modelo multidimensional, desarrolla el rol de los apoyos y propone un marco de acción para planificar e implementar apoyos que mejoran el funcionamiento (AAIDD, 2010; Verdugo, 2009).

El diseño universal para el aprendizaje (DUA), formulado por Meyer, Rose y Gordon (2014), se ha destacado como una de las buenas prácticas inclusivas que son coherentes con el marco multidimensional del funcionamiento humano (AAIDD, 2010) y el enfoque ecológico-contextual, ya que se enfoca en la modificación del contexto para posibilitar que los y las estudiantes tengan acceso al currículo. El principio fundamental que subyace a la propuesta del DUA es el de accesibilidad (Simón et al., 2016), ya que se apunta a abordar las barreras de acceso que impone un currículo inflexible. De esta forma, el DUA apunta a 
desarrollar un currículo más expansivo, variado y flexible y no más adaptado (Simón et al., 2016). Los autores defienden que un currículo diseñado de esta manera permite "eliminar las barreras innecesarias sin eliminar los desafíos necesarios" en el currículo (CAST, 2011 , p.2), de manera que los cambios, así como la dedicación de tiempo y recursos adicionales para los ajustes posteriores -o "adecuaciones curriculares"- sean innecesarios (CAST, 2011). Un rasgo esencial de esta propuesta es la oferta de opciones personalizables para el acceso al currículo, de manera que permita a todos los estudiantes progresar partiendo de su propio nivel (Meyer et al., 2014), de manera que la enseñanza permita satisfacer las necesidades de aprendices diversos al mismo tiempo que se mejora el proceso de aprendizaje de todos y todas (Capp, 2017). El DUA, tiene como finalidad última que la educación contribuya al aprendizaje generando aprendices expertos, es decir, estudiantes involucrados que dominen su propio proceso de aprendizaje (Meyer et al., 2014), lo cual es a su vez coherente con el principio de participación indicado anteriormente.

Según su formulación, una planificación desde el DUA debe reflejarse en los cuatro componentes del currículo, a saber: metas, materiales, métodos y evaluación. La fundamentación del DUA y su implementación se sustenta en tres principios centrales (de cuales se derivan nueve pautas y 31 verificadores). Estos principios apuntan a tres grandes procesos psicológicos implicados en el aprendizaje. El principio de motivación apunta a la variedad de formas en que los y las estudiantes se involucran y motivan con el contenido de la clase. El principio de representación apunta a la variedad de formas en que los y las estudiantes se representan el contenido. Por su parte, el principio de acción y expresión apunta a la variedad de formas en que los y las estudiantes pueden demostrar su acción y expresar la adquisición de conocimientos (Meyer et al., 2014).

Estos tres principios, a su vez, se despliegan en nueve procesos psicológicos (tres de cada principio) (llamados pautas en su formulación original) dentro de los cuales se sugiere la proporción de opciones que atiendan a la variedad anteriormente referida. Dentro del principio de motivación, se presentan opciones diversificadas para: i) la autorregulación; ii) mantener el esfuerzo y persistencia; iii) captar el interés. Dentro del principio de representación, se presentan opciones para: iv) la comprensión; v) el lenguaje y los sistemas simbólicos; y vi) la percepción. Finalmente, dentro del principio de acción y expresión, se presentan opciones para: vii) la función ejecutiva; viii) la expresión y la comunicación; y para ix) la acción física. En su formulación, para cada uno de estos procesos o pautas, se consideran un total de 31 indicadores o verificadores (opciones de diversificación), los cuales cuentan a su vez con ejemplos específicos de aplicación (Meyer et al., 2014).

La literatura referida a la aplicación de proyectos curriculares basados en DUA sugiere resultados que apoyan su implementación (Sánchez et al., 2019). El metaanálisis de Capp (2017) concluye que el DUA es una metodología efectiva para mejorar el proceso de aprendizaje de todos los estudiantes y que tiene efectos en mayores niveles de motivación, experiencias mejoradas de lectura, habilidad para elaborar preguntas, mejoras en el proceso de escritura, mayor disfrute al usar tecnología y mayor autonomía (Capp, 2017). Otros autores han indicado que, en el marco del DUA, el uso de herramientas basadas en tecnología ofrece apoyos cognitivos para el aprendizaje y la evaluación, y un impacto positivo en las interacciones entre estudiantes con y sin discapacidad (Rao, Wook y Bryant, 2014). Así, se ha reportado evidencia a favor del DUA como un elemento clave de la educación inclusiva, no solo a nivel de aprendizaje, sino también a nivel de participación. 
Como un aspecto importante, se ha planteado que el DUA sería un modelo alternativo y opuesto a los modelos de adecuaciones curriculares posteriores, ya que en él se deben anticipar las preferencias y necesidades de los estudiantes (Capp, 2017; Thousand, Villa y Nevin, 2015). Así, se argumenta que el profesor "primero piensa en las necesidades de los estudiantes dentro del aula, y después va al currículo" (Capp, 2017, p. 793). A pesar de esta aseveración, no ha habido un desarrollo teórico ni práctico con respecto al papel de las necesidades de los estudiantes en la planificación del currículo, es decir, cómo éstas son evaluadas, consideradas e integradas al momento de planificar.

En este sentido, el constructo necesidades de apoyo desarrollado en el marco del paradigma de apoyos (AAIDD, 2010; Schalock et al., 2010; Thompson et al., 2009) surge como un concepto teórico pertinente y esclarecedor para abordar las necesidades de los estudiantes desde el DUA. Este paradigma profundiza el rol de los apoyos señalados en el modelo de funcionamiento humano y es el marco para la provisión de apoyos que buscan mejorar el funcionamiento y resultados personales deseados de las personas con discapacidad (Schalock y Luckasson, 2013a, 2013b; Schalock et al., 2010). Ya que este paradigma se alinea con el enfoque socio ecológico o ecológico contextual, que se basa en la interacción de la persona-contexto (Verdugo, Schalock, Thompson y Guillén, 2013), el paradigma de apoyos tiene como premisa que los apoyos individualizados reducen el desajuste entre persona y su entorno (Schalock et al., 2010). Así, y en línea con en el marco comprensivo multidimensional de funcionamiento humano, se considera que los apoyos son un medio para mejorar el funcionamiento de la persona (Schalock et al., 2010).

Las bases conceptuales de este paradigma profundizan el rol de los apoyos en tres niveles, a saber: i) las necesidades de apoyo, entendidas como un constructo referido al patrón e intensidad de apoyos necesarios para que una persona participe en actividades comunes o relevantes; ii) los apoyos, que se entienden como todos aquellos recursos y estrategias para promover el desarrollo, educación, intereses y bienestar personal de un individuo y mejorar su funcionamiento; y iii) el sistema de apoyos, correspondiente al uso planificado e integrado de estrategias y recursos de apoyos individualizados (para lograr los fines antes mencionados) y que incluyen los distintos aspectos del funcionamiento humano en múltiples contextos (Schalock et al., 2010; Thompson et al, 2009).

Este enfoque proporciona una estructura para pensar sobre las funciones específicas implicadas en la prestación de apoyos (Schalock et al., 2010). Articulando sus niveles, el modelo de apoyos parte de las necesidades individuales de cada persona producto de la discrepancia existente entre las competencias personales y las demandas del entorno. Este desajuste visibiliza las necesidades de apoyo que han de ser abordadas mediante apoyos que las cubran, y esta cobertura es posible mediante la planificación y aplicación sistemática de los apoyos (Amor, 2017).

A este respecto se ha propuesto que el funcionamiento humano está influido y es potencialmente mejorable por el uso de siete componentes de un sistema de apoyos: (i) sistemas organizativos, (ii) incentivos, (iii) apoyos cognitivos, (iv) herramientas, (v) entorno físico, (vi) habilidades/conocimiento, y (vii) capacidad inherente (Schalock et al., 2010). A nivel de proceso la aplicación del paradigma de apoyos sigue una serie de pasos: (i) identificación de experiencias y metas; (ii) evaluación de necesidades de apoyo; (iii) desarrollo e implementación del plan de apoyos; (iv) supervisión del progreso; y (v) valoración de los resultados personales (Schalock, 2010; Thompson et al., 2009; Van Loon, 2009, 2015; Verdugo, Arias e Ibáñez, 2007; Verdugo et al., 2014, 2016). 
Con respecto al constructo necesidades de apoyo se ha indicado que la intensidad de éstas refleja una característica relativamente duradera y no simplemente la necesidad de un apoyo específico en un momento dado. En la evaluación de necesidades de apoyo el foco no está en lo que a la persona le dificulta, si no en qué medida cierto apoyo mejora su funcionamiento. Esta forma de evaluación puede observarse en escalas para la evaluación de intensidad de apoyos como lo es la SIS y la SIS-C (Verdugo et al., 2014). De esta forma, la provisión de apoyos debe siempre responder a una adecuada evaluación de las necesidades de apoyo de una persona. En la literatura, los apoyos que se planifican y proveen generalmente son individualizados, y han de proporcionarse con la búsqueda de una mejora tanto en el funcionamiento humano como en resultados personales deseados. A su vez, se plantea que los sistemas de apoyos deberían planificarse teniendo en cuenta todas las dimensiones del funcionamiento humano y los múltiples entornos en que la persona se desenvuelve para lograr una real mejora. Así, los apoyos tienen una doble función, por un lado, señalan la discrepancia entre lo que la persona no es capaz de hacer y qué cambios hacen posible su participación; y por otro, se centran en mejorar los resultados personales a través de la mejora del funcionamiento humano (AAIDD, 2010).

El paradigma de apoyos ha tenido un impacto significativo en el campo de la discapacidad, situándose como un elemento revolucionario, y por ello se ha reclamado una mayor aplicación del mismo en entornos escolares (Amor et al., 2018; Echeita et al., 2017). A pesar de lo anterior, y de la importancia de diseñar e implementar apoyos a edades tempranas, la mayor parte de los avances e implementaciones de sistemas de apoyos se adscriben a servicios o contextos laborales, apuntando a población adulta, y no al contex to escolar (Van Loon, 2009, 2015; Verdugo et al., 2016; Walker et al., 2014). Además, la mayor parte de los estudios que reportan planificación de apoyos en contexto escolar, consideran únicamente apoyos individualizados e individuales, centrados en estudiantes con discapacidad, y no consideran la planificación de apoyos universales (para todos los estudiantes) (Amor et al., 2018; Walker et al., 2014).

A pesar de que el DUA se ha destacado como una buena práctica inclusiva alineada con el enfoque socio ecológico y el modelo multidimensional, y que se ha destacado que su planificación debe responder de forma anticipada a las necesidades de sus estudiantes, no se ha presentado claridad sobre cómo se vincula la evaluación de las necesidades de apoyo de los estudiantes con los apoyos que se proporcionan en el marco del DUA. Es decir, si bien el DUA cuenta con un marco de acción ampliamente desarrollado en su forma de implementación, y es coherente con el paradigma de apoyos - ya que considera una serie de apoyos para modificar el contexto y así mejorar el funcionamiento de los estudiantesno existe claridad teórica ni metodológica sobre cómo se anticipan sistemáticamente las necesidades de apoyo de los estudiantes para evitar adecuaciones posteriores.

$\mathrm{Si}$ bien algunos trabajos recientes han profundizado la importancia de evaluar las necesidades de los estudiantes al momento de planificar desde el DUA, esta evaluación se ha considerado de una forma no sistematizada. Se plantea, de forma central, que las decisiones sobre como flexibilizar y proporcionar opciones de forma adecuada para los estudiantes recaen en el criterio profesional del docente sobre las necesidades, fortalezas, preferencias e intereses de sus estudiantes (CAST, 2018; Cook y Rao, 2018). Si bien el docente es el actor adecuado para evaluar las necesidades de sus estudiantes al momento de planificar la clase, es de utilidad contar con un modelo sistemático con el que estas necesidades puedan ser evaluadas de forma válida y confiable, para luego ser vinculadas pertinentemente a la planificación. 
En Chile, en particular, además de la falta de estudios nacionales sobre la puesta en práctica del DUA, la falta de claridad sobre cómo planificar con base en las necesidades de los estudiantes se vuelve especialmente relevante. Esto, a la luz de la promulgación del decreto $n^{\circ} 83 / 2015$ (MINEDUC, 2015), la cual ha sido valorada como un avance desde una perspectiva inclusiva (Duk, Cisternas y Ramos, 2019). En esta nueva normativa, que responde a la necesidad de que todos los estudiantes, independientemente del tipo de discapacidad, puedan acceder al currículo nacional, se señala que el diseño universal para el aprendizaje constituye el primer paso para responder a las diferencias individuales de los estudiantes. Ahora, es particularmente relevante fortalecer la implementación del diseño universal para el aprendizaje no sólo a la luz de su obligatoriedad, sino especialmente atendiendo a aquellos estudiantes con mayores dificultades que puedan, por normativa, quedar fuera de los beneficios del DUA. Esto, debido a que la normativa señala que

\begin{abstract}
(...) cuando las estrategias de respuesta a la diversidad basadas en el Diseño Universal de Aprendizaje no permitan responder a las necesidades de aprendizaje de algunos estudiantes, es necesario que se realice un proceso de evaluación diagnóstica individual para identificar si estos presentan necesidades educativas especiales y si requieren medidas de adecuación curricular. (MINEDUC, 2015, p. 24)
\end{abstract}

Esta afirmación de la normativa parece contradecir el principio fundamental que inspira el modelo del DUA, que es responder a las necesidades de todos los estudiantes. Incluso, cabe destacar que la normativa nombra "diseño universal de aprendizaje" en vez de "diseño universal para el aprendizaje", lo cual puede conllevar una mala comprensión del modelo al considerar que existe una "forma universal de aprender", siendo que el nombre correcto indica que es un diseño diversificado pensado de forma universal para atender las distintas necesidades de aprendizaje de todos los estudiantes. Por todo lo anterior, se vuelve crucial trabajar para que la planificación del DUA responda efectivamente a dichas necesidades de aprendizaje individuales de manera de que todos los estudiantes puedan verse favorecidos.

El objetivo del presente artículo es proponer un modelo comprensivo del DUA como un sistema de apoyos, identificando los apoyos que se derivan del mismo, y los lineamientos para la evaluación de las necesidades de apoyo en este contexto. La vinculación teórica y operacional entre ambos modelos servirá, a nivel teórico, para incorporar las necesidades de apoyo en el marco del DUA, y a nivel metodológico como un insumo para el desarrollo de instrumentos que evalúen las necesidades de apoyo de los estudiantes para la planificación de sistemas de apoyo desde el DUA.

\title{
1. DUA como una planificación de sistemas de apoyos para el aprendizaje
}

$\mathrm{El}$ argumento central que permite concebir al DUA como un sistema de apoyos es que implica una articulación planificada e integrada de estrategias y recursos de apoyos (AAIDD, 2010) que mejoran el funcionamiento o aprendizaje de todos los estudiantes, y que, por tanto, debe responder a la evaluación de las necesidades de apoyo para el aprendizaje de los mismos. Si bien tradicionalmente el concepto de apoyos se ha aplicado con más fuerza como apoyos de carácter individualizados en planificaciones y adecuaciones individuales (Van Loon, 2015; Verdugo et al., 2016; Walker et al., 2014;), el presente artículo propone la posibilidad de concebir los apoyos de forma universal (para todos), sin desligarlos de las necesidades individuales de cada estudiante. 
Dado que el concepto de apoyos viene a responder a las necesidades de apoyo de cada individuo, y así mejorar su funcionamiento individual, se ha planteado que los apoyos deben planificarse y aplicarse sistemáticamente en respuesta dichas necesidades, siempre buscando abordar la discrepancia existente entre las competencias personales y las demandas del entorno (Amor, 2017). No es distinto pensar esto desde el DUA, de manera que, como sistema de apoyos para el aprendizaje, debe responder a las necesidades de apoyo para el aprendizaje de todos los estudiantes, apuntando a disminuir la discrepancia entre éstos y su entorno, mejorando así el funcionamiento (en este caso, el aprendizaje) de todos.

De esta forma, entendiendo el DUA como un sistema de apoyos para el aprendizaje, se comprende que "proporcionar opciones" para cada principio que lo compone (Motivación, Representación y Acción y expresión) se traduce a proporcionar tipos de apoyo que pueden prestarse bajo cada principio. Una reformulación del modelo de Meyer y colaboradores (2014), ahora comprendiendo al mismo como un sistema de apoyos para el aprendizaje.

Cuadro 1. Apoyos para el aprendizaje comprendidos en el DUA en cada principio

\begin{tabular}{lll}
\hline \multicolumn{1}{c}{$\begin{array}{c}\text { APOYOS A LA } \\
\text { MOTIVACIÓN }\end{array}$} & APOYOS A LA REPRESENTACIÓN & $\begin{array}{c}\text { APOYOS A LA ACCIÓN Y } \\
\text { EXPRESIÓN }\end{array}$ \\
\hline $\begin{array}{l}\text { Apoyos a la } \\
\text { autorregulación }\end{array}$ & Apoyos para la comprensión & $\begin{array}{c}\text { Apoyos a la función } \\
\text { ejecutiva }\end{array}$ \\
\hline $\begin{array}{l}\text { Apoyos al esfuerzo y la } \\
\text { persistencia }\end{array}$ & $\begin{array}{l}\text { Apoyos para el lenguaje, las } \\
\text { expresiones matemáticas, y los } \\
\text { símbolos }\end{array}$ & $\begin{array}{l}\text { Apoyos para la expresión } \\
\text { y la comunicación }\end{array}$ \\
\hline $\begin{array}{l}\text { Apoyos para captar el } \\
\text { interés }\end{array}$ & Apoyos para la percepción & Apoyos a la acción física \\
\hline
\end{tabular}

Fuente: Propuesta propia con base en el modelo de Meyer y colaboradores (2014).

Ahora, se argumenta que el DUA correspondería a un sistema de apoyos en tanto se implementa de forma articulada diversos tipos de apoyos posibles que son coherentes a su vez con los siete componentes considerados tradicionalmente en un sistema de apoyos (apoyos organizativos, incentivos, apoyos cognitivos, herramientas, entorno físico, habilidades/conocimiento, capacidad inherente) (Schalock et al., 2010). A su vez, el DUA funcionaría como una planificación articulada de un sistema de apoyos en tanto presenta una articulación planificada de apoyos que refieren a distintos componentes del currículo (metas, materiales, método y evaluación).

De esta forma, el DUA podría conceptualizarse como la planificación de un sistema de apoyos que considera una diversidad de apoyos posibles que son agrupables en tres tipos globales de apoyo (según los principios) y los cuales se planifican en torno a cuatro componentes del currículo en los que se reflejan o implementan. Si bien en la literatura los sistemas de apoyos deberían conceptualizarse teniendo en cuenta todas las dimensiones del funcionamiento humano y los múltiples entornos para lograr una real mejora (AAIDD, 2010) se considera que desde el DUA sólo se pueden atender todas las dimensiones a las que el currículo pueda aludir dentro del aula, considerando que la aplicación del paradigma de apoyos en el acceso al currículo desde los apoyos considerados en el DUA, no cumple con la mismas características que aquellos sistemas de apoyo integrales que incorporan variadas esferas de la vida. 


\section{Necesidades de apoyo para el aprendizaje en el marco del DUA}

Como se ha fundamentado anteriormente, el principal foco de esta propuesta es fortalecer el modelo de Meyer y colaboradores (2014) incorporando el constructo de necesidades de apoyo, específicamente, el constructo necesidades de apoyo para el aprendizaje. En concreto, la propuesta implica utilizar el marco conceptual de principios y pautas como referencia para la organización de la evaluación de necesidades de apoyo que permita mejorar el diseño de apoyos específicos que favorezcan el aprendizaje y participación de todos los estudiantes. Se ha justificado que realizar esta vinculación no sólo posee coherencia a nivel teórico, sino que provee ventajas a nivel metodológico sobre el cómo se pueden evaluar, con base en indicadores observables, las necesidades de los estudiantes en el marco del DUA.

En esta propuesta, las necesidades de apoyo para el aprendizaje son entendidas como patrón e intensidad de apoyos a la motivación, representación, acción y expresión, necesarios para que un/a estudiante aprenda en aula común (junto a todos los estudiantes). A partir de ello, la evaluación de las necesidades de apoyo para el aprendizaje referida a cada principio del DUA, da por resultado la evaluación de tres dimensiones del constructo: necesidades de apoyo para el aprendizaje asociadas a la motivación, necesidades de apoyo para el aprendizaje asociadas a la representación y necesidades de apoyo para el aprendizaje asociadas a la acción y expresión.

De esta forma, las necesidades de apoyo para el aprendizaje asociadas a la motivación serán comprendidas como el patrón e intensidad de apoyos requeridos por el/la estudiante para poder implicarse en el aprendizaje según sus preferencias. Las necesidades de apoyo para el aprendizaje asociadas a la representación serán entendidas como el patrón e intensidad de apoyos requeridos por el/la estudiante para poder percibir y comprender la información que se le presenta. Finalmente, las necesidades de apoyo para el aprendizaje asociadas a la acción y expresión serán entendidas como el patrón e intensidad de apoyos requeridos por el/la estudiante para poder llevar a cabo las tareas de aprendizaje y la forma de expresar los conocimientos que se han alcanzado.

\section{Evaluación de necesidades de apoyo para el aprendizaje}

Contar con definiciones conceptuales claras del o los constructos que se desean poder evaluar es el primer paso para el desarrollo de instrumentos de medición. Una vez éstas ya han sido determinadas, como en el apartado anterior, es necesario poder operativizar dichas definiciones, es decir, establecer en qué indicadores o aspectos observables de la conducta se puede concluir la presencia de un constructo latente (Muñiz y FonsecaPedrero, 2008).

Es importante destacar nuevamente que el constructo de necesidades de apoyo ya cuenta con avances en la evaluación psicométrica del mismo, como es el caso de las escalas de intensidad de apoyos SIS y SIS-C (Verdugo et al., 2007, 2014). Para evaluar este constructo, en estas escalas se considera como principal indicador la frecuencia con que una necesidad se presenta, dicho de otra forma, la frecuencia con la que el realizar una determinada actividad requiere apoyo o puede realizarse de forma autónoma. 
En los cuadros que se presentan a continuación se expone, a modo preliminar, una propuesta de operacionalización de cada una de las dimensiones del constructo (motivación, representación y acción y expresión), sus nueve subdimensiones, y teniendo como último nivel los indicadores conductuales. Estos indicadores fueron establecidos a partir de los 31 verificadores ya considerados en el DUA.

Cuadro 2. Indicadores de necesidades de apoyo para el aprendizaje referidos al principio motivación

\begin{tabular}{|c|c|c|}
\hline PAUTAS & VERIFICADORES & INDICADOR DE NECESIDAD DE APOYO* \\
\hline \multirow{4}{*}{$\begin{array}{l}\text { Apoyos para la } \\
\text { autorregulación }\end{array}$} & \multirow{2}{*}{$\begin{array}{l}\text { Promover expectativas y } \\
\text { creencias que optimicen la } \\
\text { motivación }\end{array}$} & $\begin{array}{l}\text { El/la estudiante confía en que sus } \\
\text { aprendizajes irán mejorando. }\end{array}$ \\
\hline & & $\begin{array}{l}\text { El/la estudiante confía en que su habilidad } \\
\text { irá mejorando. }\end{array}$ \\
\hline & $\begin{array}{l}\text { Facilitar niveles graduados } \\
\text { de apoyo para imitar } \\
\text { habilidades y estrategias. }\end{array}$ & $\begin{array}{l}\text { El/la estudiante es capaz de regular sus } \\
\text { emociones en las actividades referidas al } \\
\text { aprendizaje o evaluación. }\end{array}$ \\
\hline & $\begin{array}{l}\text { Desarrollar la } \\
\text { autoevaluación y la } \\
\text { reflexión }\end{array}$ & $\begin{array}{l}\text { El/la estudiante es capaz de tolerar la } \\
\text { frustración en las actividades referidas al } \\
\text { aprendizaje o su evaluación. }\end{array}$ \\
\hline \multirow{8}{*}{$\begin{array}{l}\text { Apoyos para } \\
\text { mantener el } \\
\text { esfuerzo y la } \\
\text { persistencia }\end{array}$} & \multirow{2}{*}{$\begin{array}{l}\text { Resaltar la relevancia de las } \\
\text { metas y los objetivos. }\end{array}$} & $\begin{array}{l}\text { El/la estudiante persevera en las tareas } \\
\text { referidas al aprendizaje o evaluación hasta } \\
\text { completarlas. }\end{array}$ \\
\hline & & $\begin{array}{l}\mathrm{El} / \text { la estudiante se mantiene concentrado/a } \\
\text { durante las actividades hasta completarlas. }\end{array}$ \\
\hline & \multirow{2}{*}{$\begin{array}{l}\text { Variar los niveles de desafío } \\
\text { y apoyo }\end{array}$} & $\begin{array}{l}\text { El/la estudiante persevera en tareas que } \\
\text { percibe como difíciles de realizar. }\end{array}$ \\
\hline & & $\begin{array}{l}\text { El/la estudiante persevera en tareas que } \\
\text { percibe como fáciles de realizar. }\end{array}$ \\
\hline & \multirow{2}{*}{$\begin{array}{l}\text { Fomentar la colaboración y } \\
\text { la comunidad }\end{array}$} & $\begin{array}{l}\text { El/la estudiante colabora con sus } \\
\text { compañeros en las actividades. }\end{array}$ \\
\hline & & $\begin{array}{l}\text { El/la estudiante trabaja en grupo con sus } \\
\text { compañeros durante las actividades. }\end{array}$ \\
\hline & \multirow{2}{*}{$\begin{array}{l}\text { Proporcionar una } \\
\text { retroalimentación orientada }\end{array}$} & $\begin{array}{l}\text { El/la estudiante persevera en las tareas aun } \\
\text { cuando cometa errores. }\end{array}$ \\
\hline & & $\begin{array}{l}\text { El/la estudiante persevera en las tareas aun } \\
\text { cuando no reciba retroalimentación de su } \\
\text { desempeño. }\end{array}$ \\
\hline \multirow{6}{*}{$\begin{array}{l}\text { Apoyos para } \\
\text { captar el } \\
\text { interés }\end{array}$} & \multirow{2}{*}{$\begin{array}{l}\text { Optimizar la elección } \\
\text { individual y la autonomía }\end{array}$} & $\begin{array}{l}\text { El/la estudiante es capaz de tomar } \\
\text { decisiones con respecto a las actividades. }\end{array}$ \\
\hline & & $\begin{array}{l}\text { Al/la estudiante se le permite tomar } \\
\text { decisiones con respecto a las actividades. }\end{array}$ \\
\hline & \multirow{2}{*}{$\begin{array}{l}\text { Optimizar la relevancia, el } \\
\text { valor y la autenticidad }\end{array}$} & El/la estudiante disfruta las actividades. \\
\hline & & $\begin{array}{l}\text { El/la estudiante comprende la importancia } \\
\text { de las distintas actividades. }\end{array}$ \\
\hline & \multirow{2}{*}{$\begin{array}{l}\text { Minimizar la sensación de } \\
\text { inseguridad y las } \\
\text { distracciones }\end{array}$} & $\begin{array}{l}\text { El/la estudiante cuenta con un clima de aula } \\
\text { con pocas distracciones. }\end{array}$ \\
\hline & & $\begin{array}{l}\text { El/la estudiante cuenta con un clima de } \\
\text { apoyo y aceptación por parte de sus } \\
\text { compañeros. }\end{array}$ \\
\hline
\end{tabular}

Nota: La frecuencia de cada indicador se indica de la siguiente forma: Nunca, Rara vez, Algunas veces, Frecuentemente, Siempre.

Fuente: Elaboración propia a partir de Meyer y colaboradores (2014) y propuesta teórica desarrollada en el artículo.

Es importante considerar esta operacionalización como una propuesta preliminar sobre la forma en cómo podrían evaluarse las necesidades de apoyo para el aprendizaje en el 
contexto del DUA. Esto, ya que, para poder realizar una evaluación de necesidades de apoyo para el aprendizaje efectiva y útil, es necesario poder enmarcar cada evaluación, y por tanto cada indicador, a un contenido curricular específico, ya que las necesidades de apoyo para el aprendizaje no serán necesariamente las mismas entre distintas asignaturas, y algunos indicadores pueden volverse más pertinentes en algunos contenidos que en otros (por ejemplo, la comprensión de fórmulas matemáticas). Del mismo modo, para evaluar las necesidades de apoyo para el aprendizaje de forma adecuada, es necesario definir los indicadores en consideración del nivel educativo, esto, debido a que los contenidos y desafíos van variando. Las definiciones teóricas de cada subdimensión y de cada verificador pueden encontrarse en Meyer y colaboradores (2014).

Cuadro 3. Indicadores de necesidades de apoyo para el aprendizaje referidos al principio de representación

\begin{tabular}{|c|c|c|}
\hline Pautas & VERIFICADORES & INDICADOR DE NECESIDAD DE APOYO \\
\hline \multirow{8}{*}{$\begin{array}{l}\text { Apoyos para la } \\
\text { comprensión }\end{array}$} & \multirow{2}{*}{$\begin{array}{l}\text { Activar } \\
\text { conocimientos } \\
\text { previos }\end{array}$} & $\begin{array}{l}\text { El/la estudiante logra recuperar información previa } \\
\text { cuando se enfrenta a un nuevo aprendizaje. }\end{array}$ \\
\hline & & $\begin{array}{l}\mathrm{El} / \text { la estudiante recuerda los contenidos vistos o } \\
\text { ejercitados previamente. }\end{array}$ \\
\hline & \multirow{2}{*}{$\begin{array}{l}\text { Destacar } \\
\text { patrones, } \\
\text { características } \\
\text { fundamentales, } \\
\text { ideas principales } \\
\text { y relaciones } \\
\text { entre ellas. }\end{array}$} & $\begin{array}{l}\text { El/la estudiante logra identificar las ideas centrales a } \\
\text { partir de un texto, audio o imagen utilizada para un } \\
\text { ejercicio (ej. identificar al protagonista de un cuento o } \\
\text { los datos de un ejercicio matemático). }\end{array}$ \\
\hline & & $\begin{array}{l}\text { El/la estudiante establece relaciones entre elementos } \\
\text { a partir de un texto, audio o imagen utilizada para el } \\
\text { ejercicio. }\end{array}$ \\
\hline & \multirow{2}{*}{$\begin{array}{l}\text { Guiar el } \\
\text { procesamiento } \\
\text { de la } \\
\text { información, la } \\
\text { visualización y } \\
\text { la manipulación }\end{array}$} & $\begin{array}{l}\text { El/la estudiante comprende información proveniente } \\
\text { de un texto, audio o imagen (ej.: identificar los } \\
\text { motivos que mueven al protagonista de un cuento). }\end{array}$ \\
\hline & & $\begin{array}{l}\text { El/la estudiante logra resolver preguntas o problemas } \\
\text { asociados a la comprensión de un texto, audio o } \\
\text { imagen. }\end{array}$ \\
\hline & \multirow{2}{*}{$\begin{array}{l}\text { Maximizar la } \\
\text { memoria y la } \\
\text { transferencia de } \\
\text { información }\end{array}$} & $\begin{array}{l}\text { El/la estudiante logra recordar contenidos asociados } \\
\text { a las palabras utilizadas durante la clase. }\end{array}$ \\
\hline & & $\begin{array}{l}\text { El/la estudiante logra recordar contenidos asociados } \\
\text { a los textos, audios o imágenes utilizadas en las clases. }\end{array}$ \\
\hline \multirow{9}{*}{$\begin{array}{l}\text { Apoyos para el } \\
\text { lenguaje, las } \\
\text { expresiones } \\
\text { matemáticas, los } \\
\text { símbolos }\end{array}$} & \multirow{2}{*}{$\begin{array}{l}\text { Apoyos para } \\
\text { definir el } \\
\text { vocabulario y } \\
\text { los símbolos }\end{array}$} & $\begin{array}{l}\text { El/la estudiante comprende los significados asociados } \\
\text { a las palabras de los textos utilizados en clase. }\end{array}$ \\
\hline & & $\begin{array}{l}\text { El/la estudiante recuerda los significados enseñados } \\
\text { en clase. }\end{array}$ \\
\hline & \multirow{2}{*}{$\begin{array}{l}\text { Clarificar la } \\
\text { sintaxis y la } \\
\text { estructura }\end{array}$} & $\begin{array}{l}\mathrm{El} / \text { la estudiante distingue la estructura (sintaxis) de } \\
\text { una frase u oración (ej.: sujeto y verbo). }\end{array}$ \\
\hline & & $\begin{array}{l}\text { El/la estudiante distingue la estructura de un texto } \\
\text { (ej.: identifica y distingue los párrafos). }\end{array}$ \\
\hline & \multirow{4}{*}{$\begin{array}{l}\text { Facilitar la } \\
\text { decodificación } \\
\text { de textos, } \\
\text { notaciones } \\
\text { matemáticas y } \\
\text { símbolos }\end{array}$} & $\begin{array}{l}\text { El/la estudiante identifica los sonidos que están } \\
\text { asociados a las letras o sílabas. }\end{array}$ \\
\hline & & $\begin{array}{l}\text { El/la estudiante logra decodificar palabras } \\
\text { previamente conocidas (identificar cómo suenan y } \\
\text { cómo se leen). }\end{array}$ \\
\hline & & $\begin{array}{l}\text { El/la estudiante logra decodificar nuevas palabras } \\
\text { (identificar cómo suenan y cómo se leen). }\end{array}$ \\
\hline & & $\begin{array}{l}\text { El/la estudiante identifica notaciones de fórmulas o } \\
\text { símbolos matemáticos }\end{array}$ \\
\hline & $\begin{array}{l}\text { Promover la } \\
\text { comprensión }\end{array}$ & $\begin{array}{l}\text { El/la estudiante comparte (habla o comprende) el } \\
\text { idioma hablado por el resto de sus compañeros/as. }\end{array}$ \\
\hline
\end{tabular}




\begin{tabular}{|c|c|c|}
\hline & $\begin{array}{l}\text { entre diferentes } \\
\text { idiomas }\end{array}$ & $\begin{array}{l}\text { El/la estudiante comparte (habla o comprende) el } \\
\text { idioma utilizado en los ejercicios. }\end{array}$ \\
\hline & $\begin{array}{l}\text { Ilustrar las ideas } \\
\text { principales a }\end{array}$ & $\begin{array}{l}\text { El/la estudiante identifica las ideas relevantes } \\
\text { mediante el texto escrito. }\end{array}$ \\
\hline & $\begin{array}{l}\text { través de } \\
\text { múltiples } \\
\text { medios }\end{array}$ & $\begin{array}{l}\text { El/la estudiante comprende ideas centrales cuando } \\
\text { éstas se transmiten mediante texto escrito. }\end{array}$ \\
\hline \multirow{6}{*}{$\begin{array}{l}\text { Apoyos para la } \\
\text { percepción }\end{array}$} & $\begin{array}{l}\text { Modificar y } \\
\text { personalizar la } \\
\text { presentación de } \\
\text { la información }\end{array}$ & $\begin{array}{l}\text { El/la estudiante percibe sin dificultad la información } \\
\text { provista en los ejercicios en la misma modalidad } \\
\text { presentada a sus compañeros/as (ej.: libro de texto } \\
\text { con tamaño de letra normal, volumen medio de la voz } \\
\text { del/la profesor/a, diapositivas presentadas ante toda } \\
\text { la clase). }\end{array}$ \\
\hline & \multirow{2}{*}{$\begin{array}{l}\text { Alternativas a la } \\
\text { información } \\
\text { auditiva }\end{array}$} & $\begin{array}{l}\text { El/la estudiante logra percibir la información de tipo } \\
\text { auditiva durante los ejercicios (ej.: el/la profesor/a o } \\
\text { un/a estudiante leen un texto en voz alta). }\end{array}$ \\
\hline & & $\begin{array}{l}\text { El/la estudiante presta atención a la información de } \\
\text { tipo auditiva durante los ejercicios (ej.: cuando el/la } \\
\text { profesor/a o un/a estudiante leen un texto en voz } \\
\text { alta). }\end{array}$ \\
\hline & \multirow{3}{*}{$\begin{array}{l}\text { Alternativas a la } \\
\text { información } \\
\text { visual }\end{array}$} & $\begin{array}{l}\text { El/la estudiante logra percibir la información de tipo } \\
\text { visual durante los ejercicios (ej.: presentación en } \\
\text { diapositivas, un texto en tinta). }\end{array}$ \\
\hline & & $\begin{array}{l}\text { El/la estudiante presta atención a la información de } \\
\text { tipo visual utilizada durante los ejercicios (ej.: letra de } \\
\text { tamaño normal en un texto o en una diapositiva). }\end{array}$ \\
\hline & & $\begin{array}{l}\text { El/la estudiante comprende ideas centrales cuando } \\
\text { éstas se transmiten mediante texto escrito. }\end{array}$ \\
\hline
\end{tabular}

Fuente: Elaboración propia a partir de Meyer y colaboradores (2014) y propuesta desarrollada en el artículo.

Cuadro 4. Indicadores de necesidades de apoyo para el aprendizaje referidos al principio de acción y expresión

\begin{tabular}{lll}
\hline \multicolumn{1}{c}{ PAUTAS } & VERIFICADORES & \multicolumn{1}{c}{ INDICADOR DE NECESIDAD DE APOYO } \\
\hline & $\begin{array}{l}\text { Varios métodos } \\
\text { de respuesta }\end{array}$ & $\begin{array}{l}\text { El/la estudiante logra expresar su comprensión de los } \\
\text { contenidos por los mismos medios solicitados a sus } \\
\text { compañeros/as (ej.: prueba en lápiz y papel). }\end{array}$ \\
\cline { 2 - 3 } & $\begin{array}{l}\text { Posibilidades } \\
\text { para interactuar } \\
\text { con los } \\
\text { materiales }\end{array}$ & $\begin{array}{l}\text { El/la estudiante logra interactuar manipular } \\
\text { efectivamente los materiales usados en la clase. }\end{array}$ \\
\cline { 2 - 3 } $\begin{array}{l}\text { Apoyos a los } \\
\text { medios físicos de } \\
\text { acción }\end{array}$ & $\begin{array}{l}\text { El/la estudiante logra realizar tareas específicas a } \\
\text { través de los materiales disponibles en la clase }\end{array}$ \\
\cline { 2 - 3 } & $\begin{array}{l}\text { Integrar el } \\
\text { acceso a } \\
\text { herramientas y } \\
\text { tecnologías de } \\
\text { asistencia }\end{array}$ & $\begin{array}{l}\text { de pantallas, tablets, softwares), el/la estudiante logra } \\
\text { acceder a la información con igual efectividad que sus } \\
\text { compañeros/as. }\end{array}$ \\
\cline { 2 - 3 } & $\begin{array}{l}\text { Cuando el/la docente utiliza tecnologías de apoyo } \\
\text { para la enseñanza de contenidos (ej.: presentaciones } \\
\text { de diapositivas, uso de videos o pantallas, etc.), el/la } \\
\text { estudiante puede acceder a la información con igual } \\
\text { efectividad que sus compañeros/as. }\end{array}$ \\
\hline $\begin{array}{l}\text { Apoyos a la } \\
\text { expresión y } \\
\text { hacer fluida la } \\
\text { comunicación }\end{array}$ & $\begin{array}{l}\text { Múltiples } \\
\text { formas o medios } \\
\text { de comunicación }\end{array}$ & $\begin{array}{l}\text { Durante la clase, el/la estudiante logra expresarse y } \\
\text { comunicarse mediante la oralidad con sus pares o } \\
\text { el/la docente. }\end{array}$ \\
\cline { 3 - 3 } & $\begin{array}{l}\text { Durante la clase, el/la estudiante logra expresarse y } \\
\text { comunicarse mediante la gestualidad. }\end{array}$ \\
\hline
\end{tabular}




\begin{tabular}{|c|c|c|}
\hline & \multirow{2}{*}{$\begin{array}{l}\text { Múltiples } \\
\text { herramientas } \\
\text { para la } \\
\text { composición y la } \\
\text { construcción }\end{array}$} & $\begin{array}{l}\text { El/la estudiante logra realizar las tareas a partir de } \\
\text { los materiales dispuestos tradicionalmente durante la } \\
\text { clase }\end{array}$ \\
\hline & & $\begin{array}{l}\text { El/la estudiante logra realizar tareas específicas a } \\
\text { través de las herramientas dispuestas durante la clase }\end{array}$ \\
\hline & \multirow{3}{*}{$\begin{array}{l}\text { Incorporación } \\
\text { de niveles } \\
\text { graduados de } \\
\text { apoyo en los } \\
\text { procesos de } \\
\text { aprendizaje }\end{array}$} & $\begin{array}{l}\text { El/la estudiante logra realizar tareas específicas de } \\
\text { igual grado de dificultad que sus compañeros/as. }\end{array}$ \\
\hline & & $\begin{array}{l}\text { Las actividades realizadas en clase son percibidas por } \\
\text { el/la estudiante como realizables. }\end{array}$ \\
\hline & & $\begin{array}{l}\text { El grado de dificultad de las actividades realizadas } \\
\text { durante la clase común es adecuado para el/la } \\
\text { estudiante. }\end{array}$ \\
\hline \multirow{8}{*}{$\begin{array}{l}\text { Apoyos a la } \\
\text { función ejecutiva }\end{array}$} & \multirow{2}{*}{$\begin{array}{l}\text { Guiar el } \\
\text { establecimiento } \\
\text { de metas } \\
\text { adecuadas }\end{array}$} & $\begin{array}{l}\text { El/la estudiante logra identificar cuando una que se le } \\
\text { propone es un reto realizable o no lo es. }\end{array}$ \\
\hline & & $\begin{array}{l}\text { El/la estudiante logra distinguir entre los distintos } \\
\text { tipos de actividades según el grado de dificultad que } \\
\text { le significan. }\end{array}$ \\
\hline & \multirow{2}{*}{$\begin{array}{l}\text { Apoyar la } \\
\text { planificación y } \\
\text { el desarrollo de } \\
\text { estrategias }\end{array}$} & $\begin{array}{l}\text { El/la estudiante logra planificar y desarrollar } \\
\text { estrategias para el cumplimiento de las actividades. }\end{array}$ \\
\hline & & $\begin{array}{l}\text { El/la estudiante planifica y desarrolla estrategias para } \\
\text { el cumplimiento efectivo de las tareas solicitadas. }\end{array}$ \\
\hline & $\begin{array}{l}\text { Facilitar la } \\
\text { gestión de } \\
\text { información y } \\
\text { de recursos }\end{array}$ & $\begin{array}{l}\text { El/la estudiante logra organizar internamente la } \\
\text { información necesaria para dar cumplimiento a una } \\
\text { tarea. }\end{array}$ \\
\hline & \multirow{3}{*}{$\begin{array}{l}\text { Mejorar la } \\
\text { capacidad para } \\
\text { hacer un } \\
\text { seguimiento de } \\
\text { los avances }\end{array}$} & $\begin{array}{l}\text { El/la estudiante identifica el nivel de logro que } \\
\text { alcanza en las actividades (ej.: identifica si ha leído } \\
\text { bien o mal una palabra o párrafo). }\end{array}$ \\
\hline & & $\begin{array}{l}\text { El/la estudiante logra identificar su propio progreso } \\
\text { (ej.: identifica que está logrando realizar tareas que } \\
\text { antes no) }\end{array}$ \\
\hline & & $\begin{array}{l}\text { El/la estudiante logra regular el esfuerzo que dispone } \\
\text { a cada tarea según la dificultad que le significa (ej.: le } \\
\text { dedica mayor tiempo a una actividad que identifica } \\
\text { como más desafiante). }\end{array}$ \\
\hline
\end{tabular}

Fuente: Elaboración propia a partir de Meyer y colaboradores (2014) y propuesta teórica desarrollada en el artículo.

\section{Tipos de apoyo considerados en la planificación}

En el apartado anterior se entregaron algunas directrices psicométricas sobre cómo evaluar las necesidades de apoyo para el aprendizaje de los estudiantes desde el DUA. Ahora, teniendo en consideración el quehacer docente es necesario consensuar que un diagnóstico que indique qué dimensiones o subdimensiones arrojan mayores o menores necesidades de apoyo no es suficiente para saber cómo planificar las actividades de aprendizaje. Para ello, el modelo del DUA formulado por Meyer y colaboradores (2014) cuenta con variados ejemplos concretos sobre cómo abordar cada una de las pautas y verificadores, es decir, provee, para cada dimensión y subdimensión variados tipos de apoyos.

A modo de ejemplo, en el caso de evaluar altas necesidades de apoyo en la dimensión representación, subdimensión "apoyos a la percepción", en el modelo del DUA se alude a formas alternativas que permitan diversificar la presentación de la información para que todos los estudiantes puedan percibir la información clave. De esta forma, los apoyos 
apuntan a proporcionar la misma información desde distintas modalidades. Así, para esta subdimensión, el DUA propone ejemplos concretos de apoyos que modifiquen y personalicen la presentación de la información (ej. cambiar el tamaño del texto, de la letra o el tipo de fuente, medir el contraste entre el fondo, texto e imagen, utilizar el color como medio de información)), ejemplos de apoyos que ofrezcan alternativas a la información auditiva (ej. utilizar subtítulos, utilizar diagramas, transcripciones escritas o videos), y ejemplos de apoyos que ofrezcan alternativas a la información visual (ej. descripciones texto-voz en imágenes, gráficos y videos, objetos físicos y modelos espaciales, facilitar claves auditivas, convertir el texto digital en audio, permitir la participación de un compañero(a) para que lea el texto en voz alta) (Meyer et al., 2014). Más ejemplos concretos para cada pauta se puede encontrar en la publicación de Meyer y colaboradores (2014).

Ahora, con respecto a la planificación de un sistema de apoyo que responda a los cuatro componentes del currículo, si bien la mayor parte de los apoyos considerados en el DUA se concentran en dos de los cuatro componentes del currículo (materiales y métodos), referidos al cómo y con qué se enseña el contenido, existen algunas recomendaciones importantes referidas a los componentes objetivos (por ejemplo, en la graduación de los niveles de dificultad) y evaluación (modalidades distintas de acción y expresión). De esta forma, si bien no se presentan en igual cantidad, es posible encontrar apoyos para cada componente del currículo en todas las dimensiones, como se resume en el cuadro 5 .

Cuadro 5. Clasificación de apoyos para el aprendizaje considerados en el DUA, según principios y componentes del currículo

\begin{tabular}{|c|c|c|c|c|}
\hline \multirow[t]{2}{*}{ PRINCIPIOS } & \multicolumn{4}{|c|}{ COMPONENTES DEL CURRÍCULO } \\
\hline & Metas & Materiales & Método & Evaluación \\
\hline $\begin{array}{l}\text { Apoyos para la } \\
\text { Motivación }\end{array}$ & $\begin{array}{l}\text { Apoyos para la } \\
\text { motivación que } \\
\text { se proporcionan } \\
\text { desde las metas }\end{array}$ & $\begin{array}{l}\text { Apoyos para la } \\
\text { motivación que } \\
\text { se proporcionan } \\
\text { desde los } \\
\text { materiales }\end{array}$ & $\begin{array}{l}\text { Apoyos para la } \\
\text { motivación que } \\
\text { se proporcionan } \\
\text { desde el método }\end{array}$ & $\begin{array}{l}\text { Apoyos para la } \\
\text { motivación en } \\
\text { la evaluación }\end{array}$ \\
\hline $\begin{array}{l}\text { Apoyos para la } \\
\text { Representación }\end{array}$ & $\begin{array}{l}\text { Apoyos para la } \\
\text { representación } \\
\text { que se } \\
\text { proporcionan } \\
\text { desde las metas }\end{array}$ & $\begin{array}{l}\text { Apoyos para la } \\
\text { representación } \\
\text { que se } \\
\text { proporcionan } \\
\text { desde los } \\
\text { materiales }\end{array}$ & $\begin{array}{l}\text { Apoyos para la } \\
\text { representación } \\
\text { que se } \\
\text { proporcionan } \\
\text { desde el método }\end{array}$ & $\begin{array}{l}\text { Apoyos para la } \\
\text { representación } \\
\text { en la evaluación }\end{array}$ \\
\hline $\begin{array}{l}\text { Apoyos para la } \\
\text { acción y la } \\
\text { expresión }\end{array}$ & $\begin{array}{l}\text { Apoyos para la } \\
\text { acción y la } \\
\text { expresión que se } \\
\text { proporcionan } \\
\text { desde las metas }\end{array}$ & $\begin{array}{l}\text { Apoyos para la } \\
\text { acción y la } \\
\text { expresión que se } \\
\text { proporcionan } \\
\text { desde los } \\
\text { materiales }\end{array}$ & $\begin{array}{l}\text { Apoyos para la } \\
\text { acción y la } \\
\text { expresión que se } \\
\text { proporcionan } \\
\text { desde el método }\end{array}$ & $\begin{array}{l}\text { Apoyos para la } \\
\text { acción y la } \\
\text { expresión en la } \\
\text { evaluación }\end{array}$ \\
\hline
\end{tabular}

Fuente: Propuesta propia con base en el modelo de Meyer y colaboradores (2014).

\section{Discusión y conclusiones}

En este artículo se ha buscado atender, mediante una propuesta teórica y una preliminar propuesta operacional, cómo dar respuesta a las necesidades de todos los estudiantes en la planificación desde el DUA. Para ello, se ha propuesto la explicitación de una vinculación teórica entre el DUA y el paradigma de apoyos. Esta vinculación es novedosa, principalmente, por que las aplicaciones del paradigma de apoyos han referido tradicionalmente a la planificación y provisión de apoyos individuales, y en este caso, si 
bien se consideran necesidades de apoyos individuales, se realiza una propuesta, desde un enfoque inclusivo, que fomenta la provisión de apoyos universales.

Esta vinculación se vuelve pertinente, debido a que ambos modelos son coherentes con un enfoque inclusivo y socio ecológico, y necesario, debido a que el comprender el DUA desde el paradigma de apoyos proporciona una posible respuesta sobre cómo atender e incorporar las necesidades individuales de los estudiantes en una planificación universal. Así, mediante la comprensión del DUA como un modelo de planificación de sistemas de apoyos articulados que responden a distintos principios (motivación, representación, acción y expresión) y que como tal, debe responder a la adecuada evaluación de necesidades de apoyo de los estudiantes, obtenemos dos ventajas principales: primero, la de incorporar en el DUA el constructo de necesidades de apoyo (específicamente para este trabajo, necesidades de apoyo para el aprendizaje), el cual es un constructo latente con desarrollos previos sobre su forma de medición; y segundo, el DUA proporciona una amplia variedad de apoyos concretos para cada principio. De esta forma, esta vinculación responde a dos necesidades por parte del docente: saber qué necesitan los estudiantes y saber cómo atender dichas necesidades.

Esta propuesta se vuelve especialmente relevante en el contexto chileno -como se mencionó anteriormente, a la luz del decreto 83/2015 (MINEDUC, 2015)- ya que apunta a mejorar la comprensión de las implicancias pedagógicas de esta propuesta así como a contribuir al desarrollo de instrumentos para la planificación docente desde el diseño universal, superando la conceptualización de los ajustes posteriores (Thousand, Villa y Nevin, 2015) como la opción por defecto cuando se trata de estudiantes con mayores dificultades. Con ello, esta propuesta busca generar aportes teóricos y metodológicos que aseguren en mayor medida que dichas necesidades sean cubiertas desde apoyos universales proporcionados en aula, considerando a todos los estudiantes, con y sin discapacidad.

Ahora, con respecto a la propuesta operacional entregada, es necesario destacar que esta es una propuesta que pretende ser un aporte para el desarrollo de instrumentos que permitan la evaluación de necesidades de apoyo para el aprendizaje de los estudiantes en el marco de una planificación desde el DUA. Sin embargo, esta propuesta es preliminar ya que, en caso de querer desarrollar un instrumento para una adecuada evaluación, se considera necesario desarrollar o especificar los indicadores propuestos según contenido curricular y según nivel educativo que se pretenda evaluar. Un aspecto importante sobre dicha evaluación es el aspecto operativo. El modelo del DUA declara responder a las necesidades de todos los estudiantes del aula, y es por ello que ha surgido esta propuesta. Sin embargo, en términos operativos es difícil poder realizar una evaluación de necesidades de apoyo a cada uno de los estudiantes del aula, para luego integrar ello en la planificación de la clase. Por ello, como punto de partida, se considera que, de realizarse, estas evaluaciones deben atender en primer lugar a aquellos estudiantes que presentan más riesgo de ser excluidos del aprendizaje en aula con todos sus compañeros, bajo la premisa que el identificar aquello que es esencial para algunos estudiantes con mayores necesidades, beneficiará finalmente a toda el aula: esencial for some, good for all (Lowrey et al., 2017; Thompson et al., 2018; Verdugo et al., 2019). En efecto, Verdugo y colaboradores (2019) indican que niños con y sin discapacidad intelectual pertenecen a un mismo continuo de necesidades de apoyo. De manera simular, Thompson y colaboradores (2018) argumentan que en la medida que en el aula se utilicen múltiples enfoques instruccionales es más probable que estos beneficien a los estudiantes con discapacidades cognitivas más significativas y que, al mismo tiempo, los métodos utilizados 
tradicionalmente con estos últimos pueden ser aplicados en conjunto con profesores regulares en contextos inclusivos para beneficiar a los estudiantes sin discapacidad.

Finalmente, con respecto a los apoyos específicos que nos permitan dar respuesta a las necesidades de apoyo para el aprendizaje evaluadas, si bien el DUA presenta una gran variedad de apoyos para cada subdimensión, al igual que sucede con el desarrollo de indicadores para la evaluación, se vuelve necesario enriquecer dichos ejemplos desde contenidos curriculares y niveles educativos específicos.

Se destacan dos proyecciones principales asociadas al desarrollo de esta propuesta teórica. En primer lugar, el desarrollo de instrumentos para la evaluación de necesidades de apoyo para el aprendizaje en el marco de la planificación curricular desde el DUA, los cuales se desarrollen de forma específica para un área curricular específica (contenido/asignatura) y cierto nivel educativo. En la misma línea, la segunda proyección es el desarrollo de revisiones que permitan enriquecer los ejemplos de apoyos entregados por el modelo de Meyer y colaboradores (2014) para atender a las necesidades de los estudiantes, ya que se vuelve necesario contar con la mayor cantidad de ejemplos pertinentes para las distintas áreas curriculares y niveles educativos para que estos sean realmente útiles para los docentes.

\section{Referencias}

Asociación Americana de Discapacidades intelectuales y del Desarrollo. (2010). Discapacidad intelectual. Definición, clasificación y sistemas de apoyo. Madrid: Alianza.

Ainscow, M. y Miles, S. (2009). Desarrollando sistemas de educación inclusiva: ¿Cómo podemos hacer progresar las políticas? En C. Giné (Coord.), La educación inclusiva. De la exclusión a la plena participación de todo el alumnado (pp. 161-170). Barcelona: Horsori Editorial.

Ainscow, M., Booth, T. y Dyson, A. (2006). Improving schools, developing inclusion. Londres: Routledge. https://doi.org/10.4324/9780203967157

Albornoz, N., Silva, N. y López, M. (2015). Escuchando a los niños: Significados sobre aprendizaje y participación como ejes centrales de los procesos de inclusión educativa en un estudio en escuelas públicas en Chile. Estudios Pedagógicos, 41(4), 81-96.

https://doi.org/10.4067/S07 18-07052015000300006

Amor, A. M. (2017). Evaluación y análisis diferencial de necesidades de apoyo en alumnos con y sin discapacidad intelectual. Pozoblanco: PRODE.

Amor, A. M., Verdugo, M. A., Calvo, M. I., Navas, P. y Aguayo, V. (2018). Psychoeducational assessment of students with intellectual disability: Professional-action framework analysis. Psicothema, 30(1), 39-45. https://doi.org/10.1080/13603116.2018.1445304

Amor, A. M., Hagiwara, M., Shogren, K. A., Thompson, J. R., Verdugo, M. A., Burke, K. M. y Aguayo, V. (2018). International perspectives and trends in research on inclusive education: A systematic review. International Journal of Inclusive Education, 22, 1277-1295

Capp, M. J. (2017). The effectiveness of universal design for learning: A meta-analysis of literature between 2013 and 2016. International Journal of Inclusive Education, 21(8), 791-807. https://doi.org/10.1080/13603116.2017.1325074

CAST. (2011). Universal design for learning guidelines version 2.0. Wakeeld, MA: CAST.

CAST. (2018). Universal design for learning guidelines version 2.2. Recuperado de http://udlguidelines.cast.org 
Cook, S. y Rao, K. (2018). Systematically applying UDL to effective practices for students with learning disabilities. Learning Disability Quarterly, 14(3), 171-191. https://doi.org/10.1177/0731948717749936

Duk, C., Cisternas, T. y Ramos, L. (2019). Formación docente desde un enfoque inclusivo. A 25 años de la Declaración de Salamanca, nuevos y viejos desafíos. Revista Latinoamericana de Educación Inclusiva, 13(2), 91-109. https://doi.org/10.4067/So7 18-73782019000200091

Echeita, G., Simón, C., Márquez, C., Fernández, M. L., Moreno, A. y Pérez, E. (2017). Análisis y valoración del área de educación del III plan de acción para personas con discapacidad en la Comunidad de Madrid (2012-2015). Siglo Cero, 48(1), 51-71. https://doi.org/10.14201/scero2017485171

Lowrey, A., Hollingshead, A., Howery, K. y Bishop, J. (2017). More than one way: Stories of UDL and inclusive classrooms. Research and Practice for Persons with Severe Disabilities, 42(4), 225242. https://doi.org/10.1177/1540796917711668

Meyer, A., Rose, D. y Gordon, D. (2014). Universal design for learning. Theory and practice. Wakefield, MA: CAST Professional Publishing.

MINEDUC. (2015). Decreto Exento, $N^{\circ} 83$. Recuperado de http://especial.mineduc.cl/

Muñiz, J. y Fonseca-Pedrero, E. (2008). Construcción de instrumentos de medida para la evaluación universitaria. Revista de Investigación en Educación, 5, 13-25.

Norwich, B. y Koutsouris, G. (2017). Addressing dilemmas and tensions in inclusive education. Oxford: Oxford University Press. https://doi.org/10.1093/acrefore/9780190264093.013.154

Rao, K., Wook, M. y Bryant, B. (2014). A review of research on universal design educational models. Remedial and Special Education, 35(3), 153-166.

https://doi.org/10.1177/0741932513518980

Sánchez, S., Jiménez, D., Sancho, P. y Moreno-Medina, I. (2019). Validación de instrumento para medir las percepciones de los docentes sobre el diseño universal para el aprendizaje. Revista Latinoamericana de Educación Inclusiva, 13(1), 89-103. https://doi.org/10.4067/So71873782019000100089

Schalock, R. L. y Luckasson, R. (2013a). ¿What's at stake in the lives of people with intellectual disability? Part I: The power of naming, defining, diagnosing, classifying, and planning supports. Intellectual and Developmental Disabilities, 51, 86-93. https://doi.org/10.1352/1934-9556-5 1.2.086

Schalock, R. L y Luckasson, R. (2013b). ¿What's at stake in the lives of people with intellectual disability? Part II: Recommendations for naming, defining, diagnosing, classifying, and planning supports. Intellectual and Developmental Disabilities, 51, 94-101.

https://doi.org/10.1352/1934-9556-51.2.094

Schalock, R. L., Borthwick-Duffy, S. A., Bradley, V. J., Buntinx, W. H. E., Coulter, D. L., Craig, E.M., ... y Yeager, M. H. (2010). Intellectual disability. Definition, classification, and systems of supports. Washington, DC: American Association on Intellectual and Developmental Disabilities.

Simón, C., Echeita, G., Sandoval, M., Moreno, A., Márquez, C., Fernández, M. L y Pérez, E. (abril, 2016). De las adaptaciones curriculares al diseño universal para el aprendizaje y la instrucción: un cambio de perspectiva. Comunicación presentada en el Congreso accesibilidad, ajustes y apoyos. Universidad Carlos III, Madrid, España.

Thompson, J. R., Walker, V. L., Shogren, K. A. y Wehmeyer, M. L. (2018). Expanding inclusive educational opportunities for students with the most significant cognitive disabilities through personalized supports. Intellectual and Developmental Disabilities, 56(6), 396-411. 
Thompson, J. R., Bradley, V. J., Buntinx, W. E., Schalock, R. L., Shogren, K., Snell, M., ... y Yeager, M. (2009). Conceptualizing supports and the support needs of people with intellectual disability. Intellectual and Developmental Disabilities, 47(2), 135-146. https://doi.org/10.1352/1934-9556-47.2.135

Thousand, J., Villa R. y Nevin, A. (2015). Differentiated instruction: Planning for universal design and teaching for college and career readiness. Thousand Oaks, CA: Corwin Press.

Van Loon, J. (2009). Un sistema de apoyos centrado en la persona. Mejoras en la calidad de vida a través de los apoyos. Siglo Cero, 4O(1), 40-53.

Van Loon, J. (2015). Planes individuales de apoyo: mejora de los resultados personales. Siglo Cero, 46(1), 25-40. https://doi.org/10.14201/scero20154612540

Verdugo, M. A, Arias, B. y Ibáñez, A. (2007). Escala de intensidad de apoyos (SIS). Manual adaptación española. Madrid: TEA.

Verdugo, M. A, Arias, B., Guillén, V. y Vicente, E. (2014). Escala de intensidad de apoyos para niños y adolescentes en el contexto español. Siglo Cero, 45(1), 24-40.

Verdugo, M. A., Schalock R. L., Thompson, J. R. y Guillén, V. (2013). Discapacidad intelectual: definición, clasificación y sistemas de apoyo. En M. A. Verdugo, y R. L. Schalock (Coords.), Discapacidad e inclusión. Manual para la docencia (pp. 89-109). Salamanca: Amarú.

Verdugo, M. A, Guillén, V., Arias, B., Vicente, E. y Badía, M. (2016). Confirmatory factor analysis of the supports intensity scale for children. Research in Developmental Disabilities, 49(50), 140152. https://doi.org/10.1016/j.ridd.2015.11.022

Verdugo, M. A, Amor, A. M, Arias, V., Guillén, V., Fernández, M. y Arias, B. (2019). Examining measurement invariance and differences across groups in the support needs of children with and without intellectual disability. Journal of Applied Research in Intellectual Disabilities, 32, 1535-1548. https://doi.org/10.1111/jar.12649

Walker, V. L, DeSpain, S. N, Thompson, J. R y Hughes, C. (2014). Assessment and planning in K12 schools: A social-ecological approach. Inclusion, 2(2), 125-139. https://doi.org/10.1352/2326-6988-2.2.125

\section{Breve CV de los autores}

\section{Victoria Sánchez-Gómez}

Psicóloga por la Universidad de Chile, con Diplomado en Métodos Cuantitativos de Investigación Psicosocial, y candidata a Magíster en Psicología Educacional. Especialización y experiencia en metodología, desarrollo de instrumentos de medición (psicometría) y análisis de datos cuantitativos. Su trabajo en investigación se enfoca en educación inclusiva y discapacidad intelectual, área en la que desarrolla tanto su tesis de magíster como otros trabajos en curso, en colaboración con académicos nacionales y extranjeros. ORCID ID: https://orcid.org/0000-0001-9330-895X. Email: victoria.sanchez@uchile.cl

\section{Mauricio López}

Psicólogo por la Universidad de Chile. Doctor en Psicología Evolutiva y de la Educación por la Universidad Autónoma de Madrid. Académico del Departamento de Psicología de la Universidad de Chile en pregrado y posgrado. Ha trabajado en el diseño de programas de formación y perfeccionamiento en educación inclusiva. Su trabajo de investigación se 
ha orientado a examinar las concepciones de profesores y estudiantes sobre procesos y fenómenos como inclusión, justicia y colaboración, buscando articular los aportes de la psicología sociocultural al desarrollo de la educación inclusiva. ORCID ID: https://orcid.org/0000-0002-8288-2988. Email: m.lopez@uchile.cl. 